\title{
8
}

\section{Case Studies of Ontology for the Knowledge Intensive Engineering Framework}

\author{
Takayuki Sekiya and Tetsuo Tomiyama \\ Department of Precision Machinery Engineering, \\ The University of Tokyo \\ Hongo 7-3-1, Bunkyo-ku, Tokyo, 113, Japan. \\ Telephone: +81-3-3812-2111 (ext. 6481). Fax: +81-3-3812-8849 \\ e-mail: $\{$ sekiya,tomiyama\}@zzz.pe.u-tokyo.ac.jp
}

\begin{abstract}
Knowledge intensive engineering is a new style of engineering in which engineering knowledge is used in a flexible and integrated manner and aims at generating more added value. A Knowledge Intensive Engineering Framework (KIEF) is a computational framework for knowledge intensive engineering. To develop KIEF, we have to build a Very Large-scale Knowledge Base (VLKB) that contains a wide variety of engineering design knowledge, from commonsense knowledge about the physical world to domain specific knowledge systematized as physics theories. VLKB for KIEF has a system of fundamental concepts called "ontology." This paper describes the role of the ontology in KIEF, and validates the effectiveness of our approach through case studies.
\end{abstract}

\section{Keywords}

Ontology, Very Large-scale Knowledge Base, Knowledge intensive engineering

\section{INTRODUCTION}

Knowledge intensive engineering is a new style of engineering in which engineering knowledge is used in a flexible and integrated manner and aims at generating more added value (Tomiyama et al., 1994). A Knowledge Intensive Engineering Framework (KIEF) is a computational framework for knowledge intensive engineering (Tomiyama et al., 1996). Figure 1 shows the concept of KIEF, in which various engineering models play a crucial role to conduct innovative engineering activities over product life cycle including design, manufacturing, operation, maintenance, and recycling.

To develop KIEF, first we have to consider the problem of how to deal with a wide variety of engineering design knowledge, from commonsense knowledge about the physical world to domain specific knowledge systematized as physics theories. Developing KIEF 


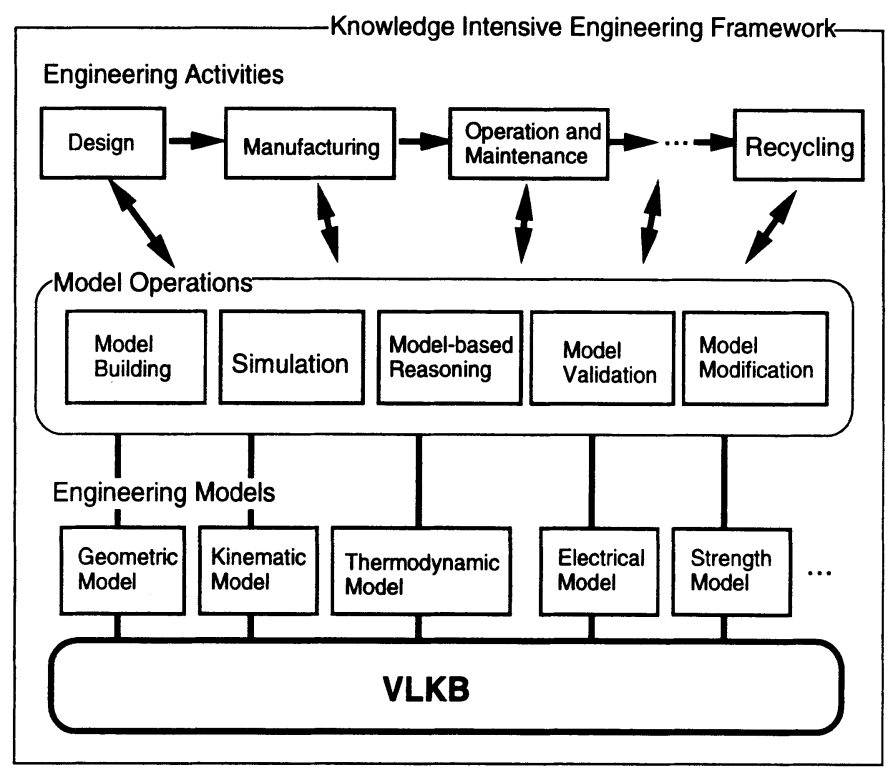

Figure 1 Knowledge Intensive Engineering Framework (Tomiyama et al., 1994)

requires to build a Very Large-scale Knowledge Base (VLKB) for dealing with a wide variety of product life cycle knowledge which is shared by participants in every stage of product life cycle (Ishii et al., 1995). However, building such VLKB is not an easy task.

VLKB for KIEF has a system of fundamental concepts called "ontology" representing knowledge about the physical world. The word, "ontology" is used with various definitions by different research groups. It is proposed as a definition for the AI community that "Ontology is a specification of a conceptualization" (Gruber, 1992a). The PACT project regards ontology as sets of agreed-upon terms and formally described meanings of design domain (Cutkosky et al., 1993). Ontology is used as a common commitment among agents which manage the design tools. Defined such, ontology is required to supply a standard vocabulary.

In our point of view, ontology generally exists independently of its expressions and representations. The knowledge available in KIEF is based on the ontology we supply, and the ontology is used for constructing a particular model and for maintaining relationships among various modelers. Therefore, the ontology provides a standard vocabulary as well as description about the physical world. In this paper, we describe the ontology for KIEF which was built and verified through our case studies. Prior to our study, there were several projects conducted towards construction of VLKB that can handle real world problems. The Cyc Project conducted at MCC (Lenat and Guha, 1989) and the How Things Work Project at Stanford University (Gruber, 1990) are examples of such projects. The Cyc Project aims at first codifying the fundamental knowledge which is common 
sense knowledge students should have before learning specific domains, such as notions of time, space, and causality. Then, the knowledge base is expected to easily acquire domain specific knowledge (even with machine learning technology). The How Things Work Project aims at collecting knowledge that explains how physical mechanisms work.

In contrast, our work does not directly aim at knowledge collection itself, but rather builds an ontology and a framework for collecting knowledge. "Knowledge collection by modeling" can be performed on our framework. In this regard, the ontology reported in this paper can be compared with Ontolingua (Gruber, 1992b).

This paper is divided into five sections. After this introduction, Section 2 briefly describes the architecture and mechanisms of KIEF. We also illustrate the framework of the ontology and discuss its roles in KIEF. Section 3 describes case studies of modeling process on KIEF. We demonstrate how to use our ontology in modeling processes, and discuss the power of our approach in collecting design knowledge. Section 4 validates the effectiveness of our approach by demonstrating the reusability of the ontology stored in the VLKB. We will observe that, even though the size of the ontology is fairly small, it is still effective. Section 5 concludes the paper.

\section{THE KNOWLEDGE INTENSIVE ENGINEERING FRAMEWORK}

KIEF aims at allowing participants in a product development process to share knowledge about the product life cycle. STEP has a similar goal to our research, but emphasizes on serving as a data model for product modeling. The aim of KIEF further extends to explanation and theory behind product models. For example, it is difficult for production engineers to understand behaviors and functions of a design object from its drawings as deep as its designers. They can, however, simply understand designers' intention, if they can perform behavior simulation of models. The purposes of this research therefore include sharing knowledge and seamless exchange of design information among various models.

A specific model, which we call an aspect model, has a specific purpose. For instance, a kinematic model simulates the kinematic behavior of the object, and a geometric model represents its shape. Therefore, a variety of models will be needed to represent a product during its life cycle. Although they represent an identical object in the real world, within the computer there is no universal interface directly connecting them, nor universal product modeling scheme. This means that, although models are practically connected with each other, it is hard to maintain or to verify the relationships among them. We consider that ontology must serve as a fundamental mechanism to maintain such relationships.

Therefore, ontology plays two crucial roles in KIEF. One is to serve as a basis of VLKB defining a wide range of knowledge including fundamental rules about physical world. The other is to provide the multiple model management mechanism with the fundamental knowledge about relationships among various aspect models.

\subsection{The Pluggable Metamodel Mechanism}

A design object can be represented with aspect models that encompass a wide variety of dimensions, i.e., domains of concepts for modeling, levels of abstraction, and levels 
of approximation. For instance, domains include function, geometry, and kinematic. A functional model represents the function, a geometric model represents the shape, and a kinematic model represents the motion of the design object. A beam model and a finite element model are used to evaluate strength at different levels of abstraction. A kinematic model with or without friction is used to evaluate motion at different levels of approximation. These aspect models are not independent of each other. Therefore, we need a mechanism to maintain the consistency among aspect models.

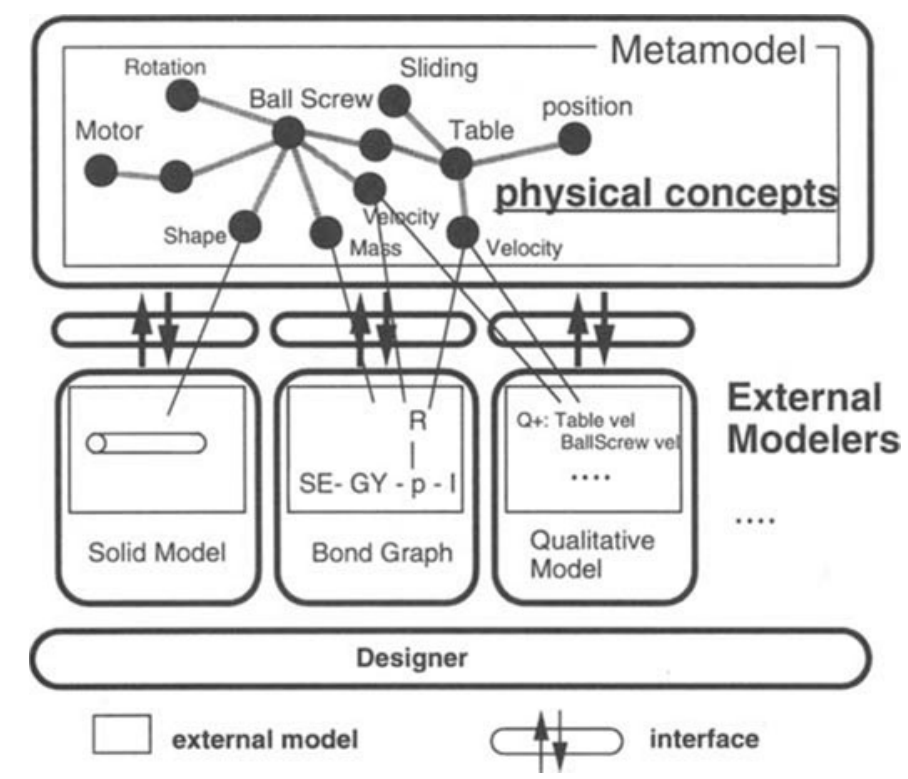

Figure 2 The pluggable metamodel mechanism (Ishii et al., 1995)

The metamodel mechanism is a modeling framework to integrate multiple aspect models (Tomiyama et al., 1989, Kiriyama et al., 1991). It has symbolic representation of concepts about physical phenomena and components (See Figure 2). A metamodel of a design object is represented as a network of relationships among concepts that appear in the aspect models. Types of relationship include causal dependency among physical phenomena, arrangements of components, and quantitative relationships. These concepts and relationships constitute the ontology of KIEF.

An aspect model is, however, usually dealt with by an existing external modeler. Therefore, it is desirable if we could plug in such existing modelers to KIEF. The pluggable metamodel mechanism allows easily plugging in external modelers (Yoshioka et al., 1993). In order to plug an external modeler into a pluggable metamodel mechanism, three kinds of knowledge have to be prepared. One is descriptions about concepts which are used by an external model. The second is descriptions about concepts which are available from 
other modelers through the metamodel mechanism. The third is descriptions about correspondences among concepts in the metamodel and model fragments used to construct an external model and procedures to translate between a concept in the metamodel and one in a model fragment. A model fragment is stored in the model libraries and used by an external modeler to build an external model. While more details are explained in the next section, here we just point out that concepts included in the these three kinds of knowledge and defined as the ontology.

\subsection{The Architecture of VLKB}

VLKB for KIEF should support model-based reasoning mechanisms, and supply fundamental knowledge about the physical world. Figure 3 depicts the architecture of VLKB to do so. VLKB consists of three layers. In the middle layer, there is a concept base which contains concepts about entities, physical phenomena, relations, and attributes. The upper layer contains physical features that are combinations of physical concepts. The lower layer contains physical laws that are used as model fragments for various aspect models (Ishii et al., 1995).

The concept base contains names of physical concepts and relationships among physical concepts. The relationships include an abstract-concrete hierarchy of physical concepts, differential relations between attributes, and "has" relations between entities and physical properties.

In the upper layer, physical features are built and stored in the physical feature knowledge base. The concept base provides only vocabulary of physical concepts such as "burn," "fuel," "warm up," and "air." Physical features are the statements that describe physical situations, such as "fuel burns, generates heat, and warms up air." The designer constructs a metamodel with physical features; that is, physical features are building blocks of a metamodel. This idea is largely based on Forbus's Qualitative Process Theory (Forbus, 1984).

In the lower layer, physical laws are described. The same physical law can be differently represented in different aspect models. The physical rule $K B$ contains unique names of physical laws and the attribute concepts used in the laws. The names of physical laws keep track of the same physical law represented in different aspect models.

The model libraries store typical model fragments of external model, which correspond to physical concepts. For example, an equation

$f=m a$

is a fragment of a dynamics equation model that describes Newton's Second Law. These fragments are used as building blocks to generate external models. To generate an external model, first relevant concepts are collected to form an aspect model in the metamodel level. The parameters used in a fragment correspond to attribute concepts, so after an aspect model is generated, the metamodel mechanism translates the concepts in the aspect model into the terminology of the corresponding external model and maintains relationships among the attribute concepts in the metamodel and the parameters in the external model. 


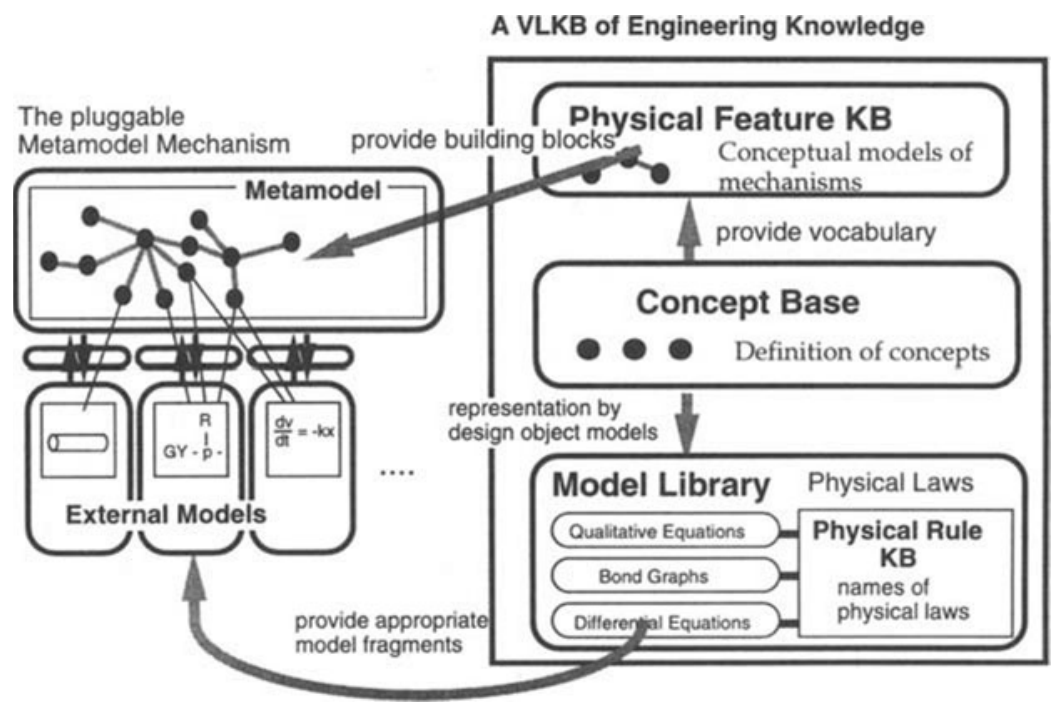

Figure 3 The Architecture of VLKB (Ishii et al., 1995)

\subsection{The Ontology for KIEF}

We consider that ontology is a system of the fundamental concepts about the physical world. The ontology for KIEF is stored in the concept base described in the previous section. It includes five kinds of concepts; namely entities, relations, attributes, physical properties, and physical phenomena. Each of these concepts is separately categorized and forms a static conceptual network.

\section{- Entity}

An entity represents an atomic, physical object in our representation. Entities are organized in an abstract-concrete hierarchy. For example, a "worm gear" is a subclass of a "gear." This implies that physical phenomena that occur on a gear pair also occur on a helical gear pair. The hierarchy allows multiple inheritance.

\section{- Relation}

A relation represents a relationship among entities, such as "on," "above," "below," and "connection." In other words, it defines the structure among entities.

- Attribute

An attribute is a concept attached to entities and takes a value to indicate the state of entities, such as "position," "temperature," and "mass."

- Physical Property

A physical property is a concept that describes a generic characteristic of entities such 
as "elastic" and "magnetized." A physical property is associated with a set of attributes that indicates degree of the physical property.

- Physical Phenomenon

A physical phenomenon designates physical laws or rules that govern behaviors. A physical phenomenon is defined by the following slots:

- Name of the physical phenomenon.

- Super (or abstract) physical phenomena.

- Prerequisites for the physical phenomenon to be activated in terms of entities together with their attribute definitions, relations, and physical properties.

- Physical laws that define relationships among the parameters and define influences that can happen when the physical phenomenon is activated.

\section{CASE STUDIES}

Building VLKB is labor- and resource-consuming. To validate our ideas, we need to evaluate burdens to build VLKB and to define and collect ontology for practical applications. More importantly, if the constructed ontology is not reusable, the whole idea is not useful. The section discusses this issue through two case studies, i.e., modeling a motorcycle and a contactor. Each case study proceeds with the following steps.

1. Build a metamodel.

2. Build a solid model.

3. Derive a set of physical equations that represents relevant physical phenomena.

In the case studies, we plugged a dynamics modeler and a solid modeler into our pluggable metamodel mechanism. The dynamics modeler is built on Mathematica* to assist a designer to derive motion equations from the metamodel and to evaluate them. We also use DESIGNBASE ${ }^{\dagger}$, which is a commercial solid modeler.

\subsection{The Motorcycle Case Study}

\section{Building a Metamodel}

First, the designer inputs a primary model of a motorcycle with physical features. A primary model represents a mental model of the designer and serves as an input to the metamodel mechanism to generate a metamodel. The designer selects physical features from the physical feature knowledge base to express the motorcycle. He/she doesn't have to deal with physical concepts directly, if the concept base has sufficient ontology.

We suppose that the motorcycle consists of two wheels, two dampers, two springs, and one body (See Figures 4 and 5). At each connection between two parts, force is transmitted, and these parts never separate. We call such connection "ForceFixedConnection" in this case study. The behavior of a motorcycle is expressed by several physical phenomena, such as "Damping," "ElasticTransformation," "LinearMotion," and "RotationalMotion."

\footnotetext{
* Mathematica is a trademark of Wolfram Research, Inc.

$\dagger$ DESIGNBASE is a trademark of RICOH Corporation.
} 


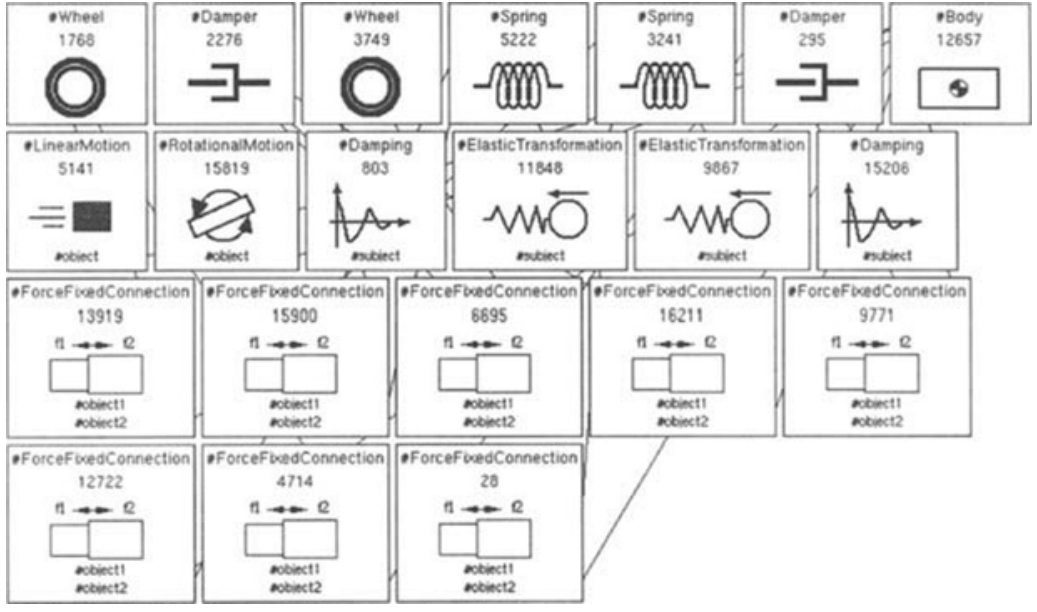

Figure 4 The Primary Model of a Motorcycle

After building the primary model, the system generates a metamodel from the primary model. This process is divided into two sub-processes. First physical phenomena are added to the primary model, which the system finds out occurring to the primary model but the designer did not notice while building the primary model. The metamodel mechanism performs this process by pattern matching between the primary model and physical features. Second, attributes are instantiated by examining the definition of each of physical phenomena which form the primary model. Through this process, relationships among attributes are also instantiated. In this example, some attributes such as "position," "velocity," "force," and some relationships among attributes such as Newton's Law (which gives the description that there are relationships among "position," "mass," and "acceleration") are instantiated and added to the metamodel of a motorcycle.

Now, the metamodel contains part of the concepts that define the motorcycle, relations among them, attributes that are used to express the behavior of the motorcycle.

\section{Building a Solid Model}

Next, the designer builds a solid model in Figure 5. Independent of the metamodel, the designer defines shape of each component, and then he/she makes correspondences between an entity concept in the metamodel and a solid. Figure 6 is a screen hardcopy of the "matching interface" used to match a solid with an entity concept, and parameters in each solid with an attribute concept. For example, the front wheel shown in the Figure 5 corresponds to an entity concept, Wheel, in the metamodel, and the position of the solid corresponds to an attribute concept, Position, of Wheel. The solid modeler obtains the position of a front wheel from the metamodel mechanism using the attribute concept as the key. When the designer builds a model independent of the metamodel as in this 


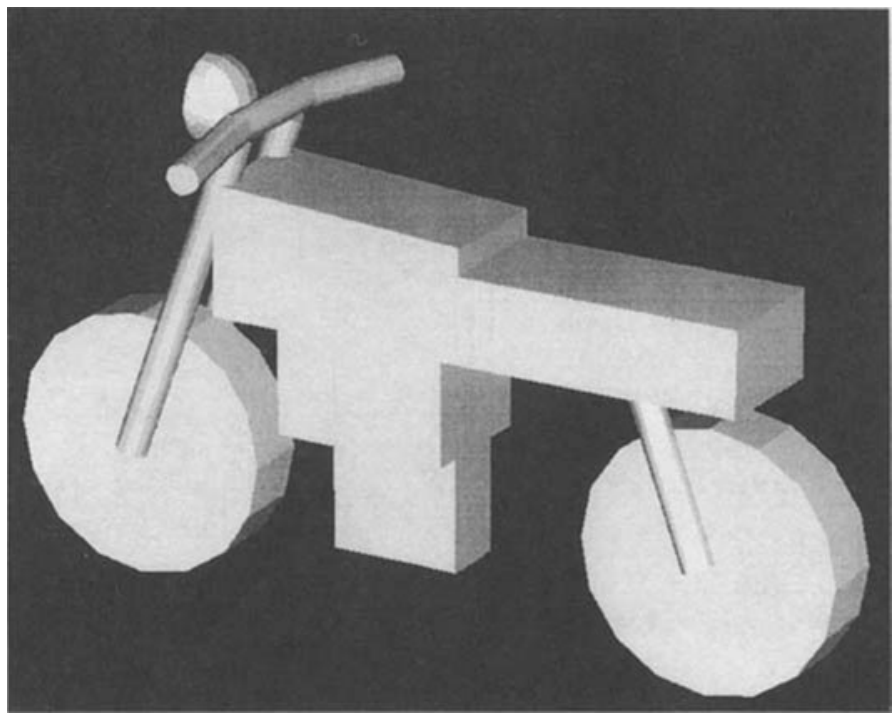

Figure 5 A Solid Model of the Motorcycle

case, he/she has to define the correspondence between a concept in the metamodel and an element of an aspect model.

\section{Building Motion Equations}

In the metamodel, the behavior of motorcycle is represented by physical phenomena, and each phenomenon is defined in VLKB using physical laws. When an external modeler composites an external model, it checks the relationships among concepts in the metamodel. Figure 7 shows the hardcopy of the dynamics modeler. The conceptual network in the upper half of this figure represents attributes and the possible relations among the attributes about the motorcycle, which are a part of the metamodel. The designer interacts with this modeler through the lower half, examines the equations the modeler gives (the lower left lists), and modifies them if necessary. The lower right lists attributes existing in the equations. The work flow to set up equations is as follows.

\section{Setting parameters.}

The dynamics modeler prepares parameters that can be used for equations by instantiating each parameter from the corresponding attribute in the metamodel. In this way, another modeler obtains the value of a parameter calculated by the dynamics modeler by simply accessing the corresponding attribute concept in the metamodel to the parameter.

2. Building physical equations.

The metamodel mechanism stores attributes and relationships among attributes, such 


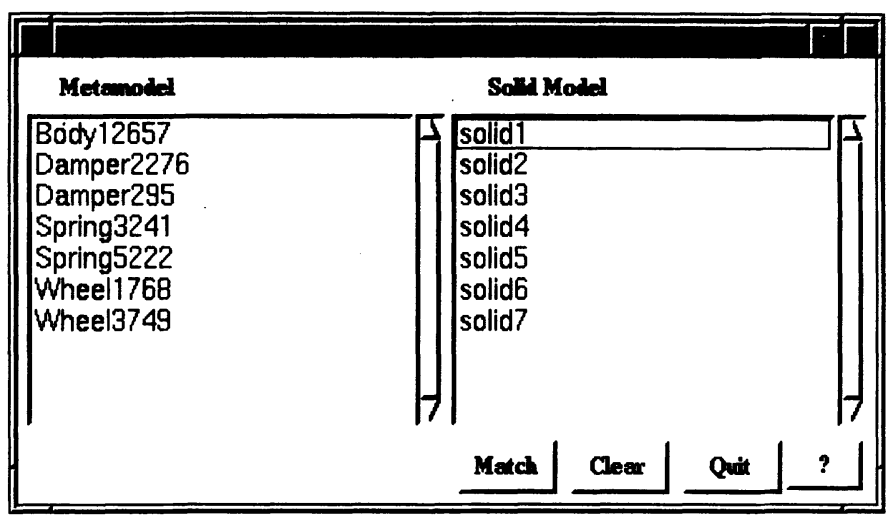

Figure 6 The Interface for Matching a Solid with an Entity Concept: "Body12657" designates the motorcycle body which is "solid1" in the solid model.

as physical laws. The model library for the dynamics modeler contains quantitative expressions of the physical laws, so the modeler replaces a physical law with a quantitative expression using this knowledge. For example, the dynamics modeler translates "Hooke's Law" expressed in the metamodel into an equation

$f=-k x$.

Given "Second Law of Newton's Laws," the process is a little more complex. The modeler knows the equation

$F=m a$,

which describes Newton's Law, and $F$ is resultant force that is defined in the concept base. When the dynamics modeler finds Newton's Law in the metamodel, it searches attributes whose names are "force" and are attached to the entity connected with the law. Then, the modeler builds an equation such as

$$
f_{1}+f_{2}+\ldots+f_{n}=m a
$$

by combining all the relevant forces.

3. Modifying the equations.

The designer modifies or arranges the given equations if necessary, because the metamodel mechanism currently cannot deal with spatial information of objects perfectly. Then the designer confirms the obtained equations.

4. Building the equations.

The modeler passes the motion equations to Mathematica, which first simplifies the 


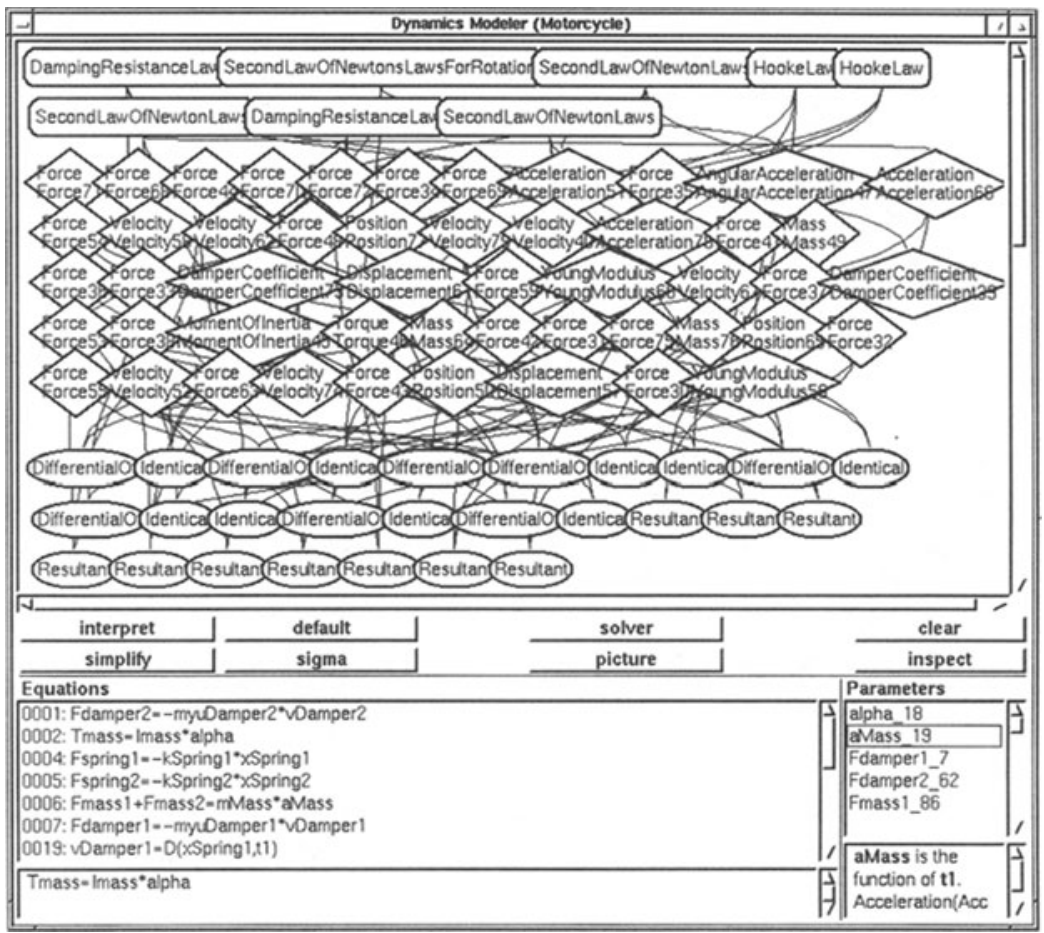

Figure 7 A Hardcopy of the Dynamics Modeler

equation. Figure 8 shows the equations (hand-assembled and simplified for the sake of readability).

\section{Solving the Motion Equations}

Next, the metamodel mechanism supplies the values of the parameters in the obtained equations, for example, from the geometric modeler. However, some values (such as viscosity) cannot be obtained in this way. In this case, the designer specifies the values. Then, Mathematica solves the equations. Figure 9 is a solution graph obtained by Mathematica. It shows the displacement and the rotation of the center of gravity of the motorcycle body, assuming that the motorcycle is running on a washboard.

The dynamics modeler can then give back the solution to the solid modeler for visual simulation. Figure 10 is the screen hard copies of the behavior simulation of the motorcycle. This is obtained in a simulation process depicted in Figure 11. 


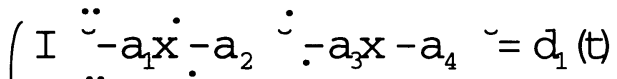

$$
\begin{aligned}
& \mathrm{m} \ddot{\mathrm{x}}-\mathrm{b}_{1} \dot{\mathrm{x}}-\mathrm{b}_{2} \sim-\mathrm{b}_{3} \mathrm{x}-\mathrm{b}_{4}=\mathrm{a}_{2}(t) \\
& a_{1}=c_{1} I_{1}-c_{2} I_{2} \quad a_{2}=-c_{1} I_{1}^{2}-c_{2} I_{2}^{2} \\
& \mathrm{a}_{3}=\mathrm{k}_{1} 1_{1}-\mathrm{k}_{2} 1_{2} \quad \mathrm{a}_{4}=-\mathrm{k}_{1} 1_{1}^{2}-\mathrm{k}_{2} 1_{2}^{2} \\
& \mathrm{~d}_{1}(t)=-\mathrm{k}_{1} 1 \mathrm{f}(\mathrm{t})-\mathrm{C}_{1} 1 \mathrm{f}(\mathrm{t})+\mathrm{k}_{2} \frac{1}{2} \mathrm{~g}(\mathrm{t})+\mathrm{C}_{2} \frac{1}{2} \mathrm{~g}(\mathrm{t}) \\
& \left.\mathrm{b}_{1}=-\mathrm{C}_{1}-\mathrm{C}_{2} \quad \mathrm{~b}_{2}=\mathrm{C}_{1}\right]_{1}-\mathrm{C}_{2} \frac{1}{2} \\
& \mathrm{~b}_{3}=-\mathrm{k}_{1}-\mathrm{k}_{2} \quad \mathrm{~b}_{4}=\mathrm{k}_{1} \frac{1}{1}-\mathrm{k}_{2} \frac{1}{2} \\
& \mathrm{~d}_{2}(t)=\mathrm{k}_{1} \mathrm{f}(\mathrm{t})+\mathrm{C}_{1} \mathrm{f}(\mathrm{t})+\mathrm{k}_{2} \mathrm{~g}(\mathrm{U})+\mathrm{C}_{2} g(t)
\end{aligned}
$$

Figure 8 Motion Equations of the Motorcycle

File $\Gamma \underline{\text { view }} \Gamma$ sound $\Gamma$

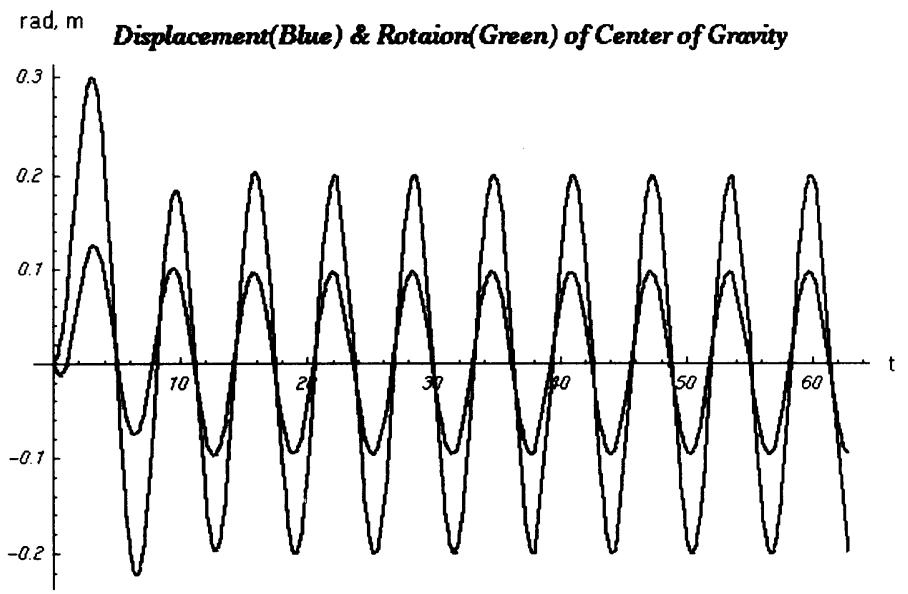

Figure 9 A Graph of the Solution 


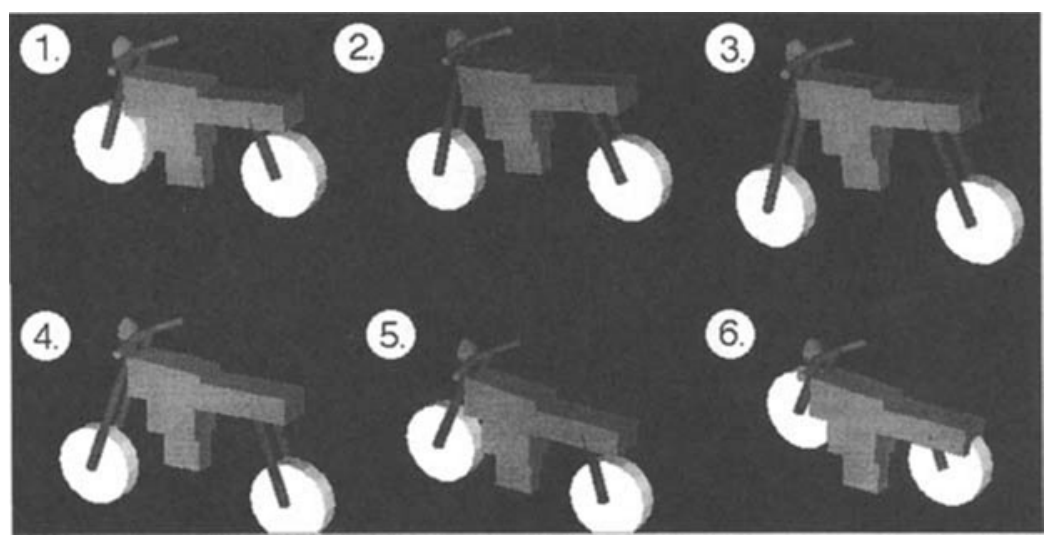

Figure 10 A Behavior Simulation

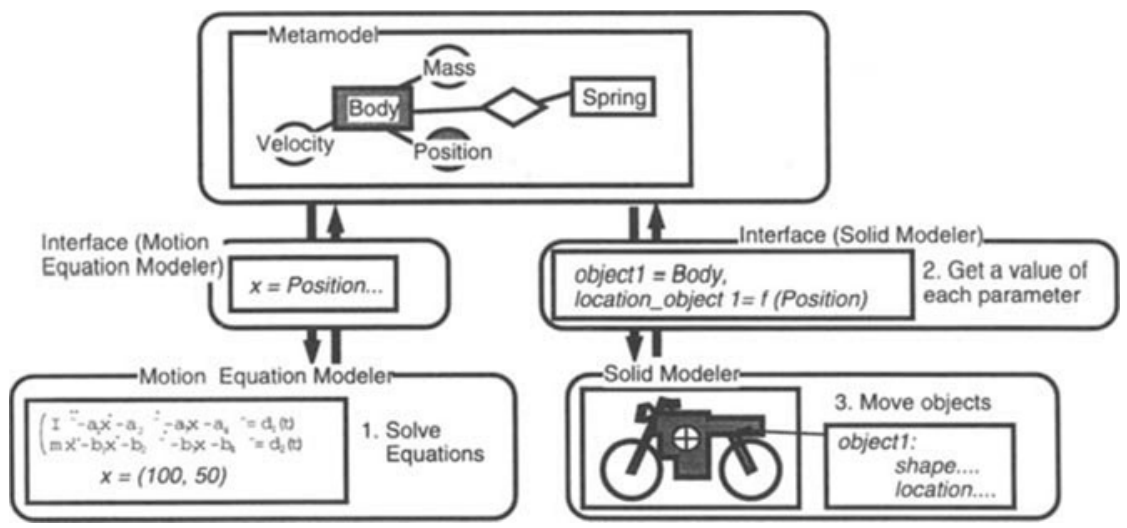

Figure 11 The Simulation Processes

\subsection{Modeling a Contactor}

We also modeled a contactor, which is a kind of electromagnetic switches. Figure 12 depicts a rough sketch of the contactor (Ranta et al., 1995). We constructed a primary model (see Figure 13). The modeling process of the contactor is not different from that of the motorcycle, so we skip the details. 


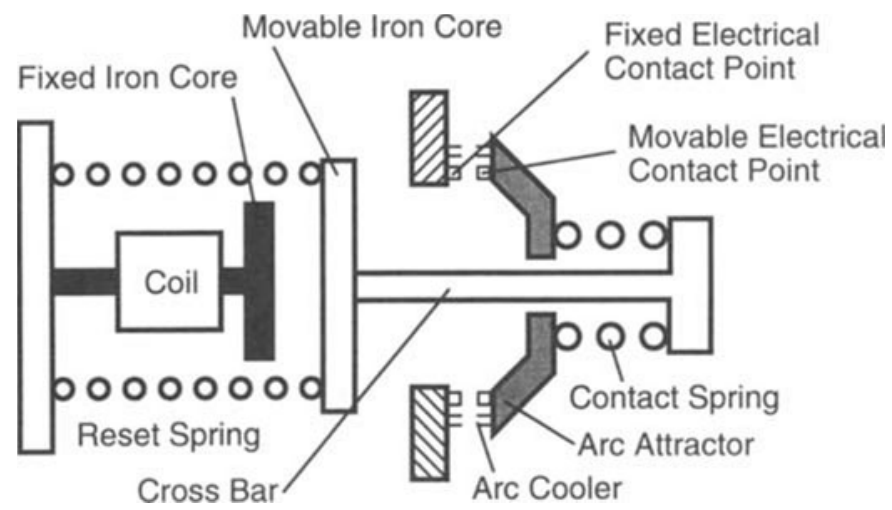

Figure 12 A Contactor

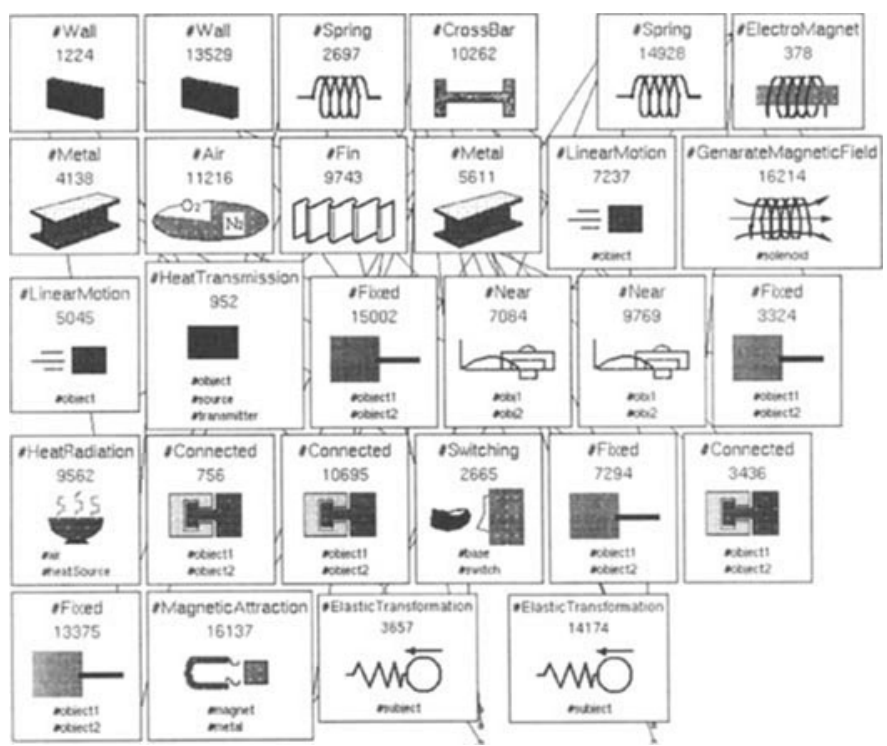

Figure 13 The Primary Model of a Contactor 


\section{DISCUSSIONS}

In this section, we validate the effectiveness of our approach by demonstrating the reusability of the ontology stored in the concept base. The concepts used for the case studies in Section 3 are not specialized to particular models, and they are reusable enough for modeling various kinds of design objects. Figure 14 shows the partial hierarchy of the entity concepts. The names circled, "Body," "Damper," "Spring," and "Wheel" are used to model the motorcycle. The names surrounded in a rectangle are used to model the contactor. Although the motorcycle and the contactor are substantially different, "Spring" is used by the both (See Figure 14). One reason for this is that in the primary design stage, concrete concepts are not used to represent the design object, because the designer cannot define its details yet.

The system can define multiple inheritance which increases the reusability of the ontology. Sub-concepts inherit the character of their super-concepts. This signifies that "the phenomenon which occurs to a super-concept will occur to its sub-concepts." For example, physical phenomenon named "LinearMotion" occurs to both "Body" of the motorcycle and "Cross Bar" of the contactor, because their super-concepts include the same concept "Mass."

Of course in the detailed modeling stage, the designer may no longer use general concepts such as "Spring," but use more concrete concepts. These concrete concepts are more specialized to the modeling object and less reusable. Note that, the motion equation deals with a "Damper," rather than "Air Damper" or "Oil Damper." (Strictly speaking, the behavior of a damper may be quite different depending on what is contained in its cylinder.) That means the difference between the two concepts are not concerned on this level of modeling, but the definition of "Damper" which is their super-concept is the matter and was useful for the case studies. This resulted in fewer concepts for modeling than we had initially imagined.

We have collected physical concepts from literature such as engineering textbooks (e.g. (Hix and Alley, 1958)), but did not need so many for modeling of the case studies. Table 1 shows the numbers of concepts we have collected, and the numbers of concepts we used or we referred to for the case studies. For example, we have collected about 250 entities, but 8 kinds of entities were used to model the motorcycle, 14 kinds of entities were used for the contactor, and 5 kinds of them were commonly used. Despite the fairly small size of the knowledge base, it sufficed for the modeling processes. This indicates the reusability of our ontology, and at the same time implies that knowledge collection for KIEF can be done during modeling processes.

We summarize the advantages of having ontology as follows. First, because the ontology supplies various engineering models with common concepts, models can share information about objects, such as quantitative values. Second, the ontology provides general concepts, like standard parts of machines. Third, the ontology supplies the abstract-concrete hierarchy that increases the reusability of knowledge.

Through the case studies, we experienced some problems. Among others, the most critical was the lack of spatial concepts. In order to treat spatial information on the metamodel, we have to systematize spatial concepts and to implement more convenient interactive tools. 


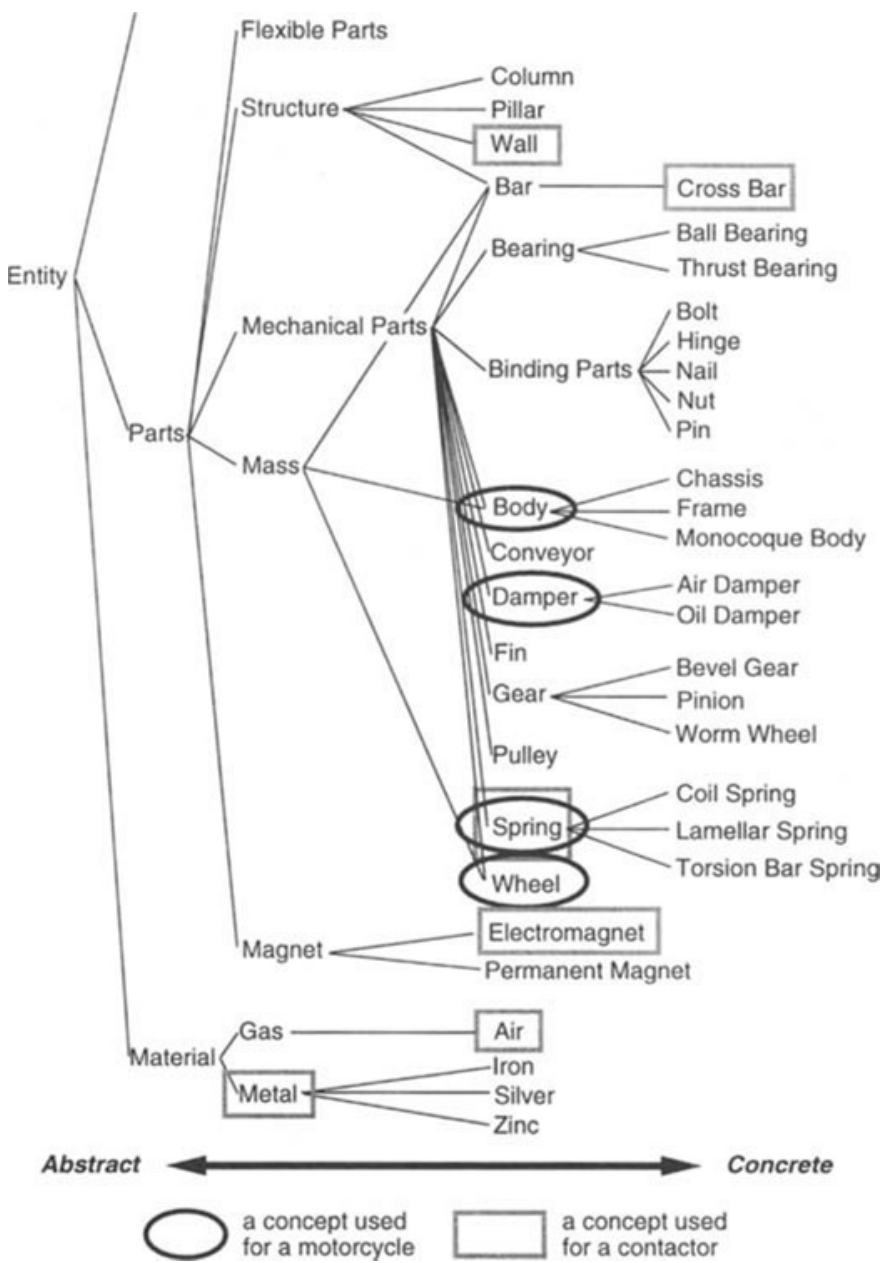

Figure 14 Part of an Entity Hierarchy 
Table 1 The Number of Concepts Used for the Case Studies

\begin{tabular}{|l|c|r|r|r||l|}
\hline \multirow{2}{*}{ Physical concept } & \multirow{2}{*}{$\begin{array}{c}\text { Total Number of Concepts } \\
\text { in the Concept Base }\end{array}$} & \multicolumn{2}{|l|}{ Concepts used for } & \multirow{2}{*}{ Source } \\
\cline { 3 - 5 } & 150 & 4 & 7 & 2 & a text book about tools and machine \\
\hline \hline Physical Phenomenon & 250 & 8 & 14 & 5 & and some examples of real products \\
\hline Entity & 40 & 1 & 4 & 1 & mainly spatial relations \\
\hline Relation & 280 & 11 & 13 & 7 & text books of dynamics, kinematics, etc \\
\hline Attribute & 80 & 3 & 6 & 2 & text books of dynamics, kinematics, etc \\
\hline Physical Property & 300 & 4 & 6 & 2 & "Physical Laws and Effects" \\
\hline Physical laws & & & Both & & \\
\hline
\end{tabular}

\section{CONCLUSIONS}

This paper first introduced the concepts of KIEF and discussed the roles of ontology in KIEF. Ontology is needed to define fundamental knowledge for KIEF and to allow knowledge sharing within the multiple model environment of KIEF. We then described the ontology for KIEF. To validate the idea of our approach, we conducted two case studies to model a motorcycle and a contactor. Each case study involved building a metamodel, a solid model, and a dynamics model. This demonstrated the power of KIEF which allows flexible knowledge sharing and reuse among various engineering models.

We collected physical concepts from literature such as engineering textbooks, but the concepts needed for the case studies were fewer than we had imagined. This suggests that collecting knowledge for KIEF can even be done through modeling processes.

\section{ACKNOWLEDGMENTS}

The work described in Section 3 was part of the international collaborative research project, GNOSIS (knowledge systematization), in the Intelligent Manufacturing Systems (IMS) research program. The example of contactor was developed under the collaboration with Helsinki University of Technology and Mitsubishi Electric Co., Ltd (Ranta et al., 1995). The authors would like to thank Dr. Yasushi Umeda, Dr. Masaharu Yoshioka, and other colleagues in our group for helping us in the discussion and the implementation of the system.

\section{REFERENCES}

Cutkosky M. R., Engelmore R. S., Fikes R. E., Genesereth M. R., Gruber T. R., Mark W. S., Tenenbaum J. M., and Weber J. C. (1993). Pact: An experiment in integrating concerrent engineeering systems. IEEE Computer, Vol. 26, No. 1, pp. 28-37, 1993.

Forbus K.D. (1984). Qualitative process theory. Artificial Intelligence, Vol. 24, No. 3, pp. 85-168, 1984.

Gruber T. (1990). The development of large, shared knowledge-bases: Collaborative activities at stanford. Technical Report KSL90-62, Stanford Knowledge Systems Laboratory, 1990. 
Gruber T. (1992). A mechanism to support portable ontologies. Technical Report KSL9166, Stanford Knowledge Systems Laboratory, 1992.

Gruber Thomas R. (1992). Ontolingua: A mechanism to support portable ontologies. Technical report KSL91-66, Knowledge Systems Laboratory, Stanford University, Stanford, 1992.

Hix C.F. and Alley R.P. (1958). Physical Laws and Effects. John Wiley \& Sons, New York.

Ishii M., Sekiya T., and Tomiyama T. (1995). A very large-scale knowledge base for the knowledge intensive engineering framework. In Mars N.J.I., editor, KBE์KS'95, the Second International Conference on Building and Sharing of Very Large-Scale Knowledge Bases, pp. 123-131, Amsterdam, Oxford, Tokyo, Washington, DC, 1995. IOS Press, Ohmsha.

Kiriyama T., Tomiyama T., and Yoshikawa H. (1991). The use of qualitative physics for integrated design object modeling. In Design Theory and Methodology (DTM '91), pp. 53-60. ASME, New York, 1991.

Lenat D.B. and Guha R.V. (1989). Building Large Knowledge-Based Systems. AddisonWesley, Reading, MA.

Ranta M., Mantyla M., Umeda Y., and Tomiyama T. (1995). Integration of functional and feature-based product modeling. Computer Aided Design, Vol. 28, No. 5, pp. 371-381, 1995.

Tomiyama T., Kiriyama T., Takeda H., and Xue D. (1989). Metamodel: A key to intelligent CAD systems. Research in Engineering Design, Vol. 1, No. 1, pp. 19-34, 1989.

Tomiyama T., Kiriyama T., and Umeda Y. (1994). Toward knowledge intensive engineering. In Fuchi K. and Yokoi T., editors, Knowledge Building and Knowledge Sharing. Ohmsha, Tokyo, Osaka, and Kyoto, 1994.

Tomiyama T., Umeda Y., Ishii M., and Yoshioka M. (1996). Knowledge systematization for a knowledge intensive engineering framework. In T. Tomiyama M. Mäntylä and Finger S., editors, Knowledge Intensive CAD Volume 1, Proceeedings of the First IFIP WG 5.2 Workshop on Knowledge Intensive CAD, pp. 33-52. Chapman \& Hall, 1996.

Yoshioka M., Nakamura M., Tomiyama T., and Yoshikawa H. (1993). A design process model with multiple design object models. In Design Theory and Methodology (DTM '93), pp. 7-14, New York, 1993. ASME.

\section{BIOGRAPHY}

Takayuki Sekiya is a Master course student in the Department of Precision Machinery Engineering, the University of Tokyo. His research interest includes model generation, model operation, and knowledge intensive engineering.

Dr. Tetsuo Tomiyama has been Associate Professor at the Department of Precision Machinery Engineering, the University of Tokyo, since 1987. From 1985 to 1987, he worked at the Center for Mathematics and Computer Science in Amsterdam. He received his doctor's degree in precision machinery engineering from the Graduate School of the University of Tokyo in 1985. His research interest includes design theory and methodology, knowledge intensive engineering, applications of qualitative physics, large scale engineering knowledge bases, and soft machines (self-maintenance machines and cellular machines). He is a member of the IFIP Working Group 5.2. 